\title{
X-Ray Standing Wave Analysis of the Effect of Isotopic Composition on the Lattice Constants of $\mathrm{Si}$ and $\mathrm{Ge}$
}

\author{
E. Sozontov, ${ }^{1}{ }^{*}$ L. X. Cao, ${ }^{1,2}$ A. Kazimirov, ${ }^{3}$ V. Kohn,${ }^{4}$ M. Konuma,${ }^{1}$ M. Cardona,${ }^{1}$ and J. Zegenhagen ${ }^{1,2, \dagger}$ \\ ${ }^{1}$ Max-Planck-Institut für Festkörperforschung, Heisenbergstrasse 1, D-70569 Stuttgart, Germany \\ ${ }^{2}$ European Synchrotron Radiation Facility, BP 220, F-38043 Grenoble Cedex, France \\ ${ }^{3}$ Cornell High Energy Synchrotron Source, Wilson Laboratory, Cornell University, Ithaca, New York 14853 \\ ${ }^{4}$ Russian Research Center "Kurchatov Institute," 123182 Moscow, Russia
}

(Received 7 February 2001)

\begin{abstract}
The $\mathrm{x}$-ray standing wave (XSW) technique is used to measure the isotopic mass dependence of the lattice constants of $\mathrm{Si}$ and Ge. Backreflection allows substrates of moderate crystallinity to be used while high order reflection yields high accuracy. The XSW, generated by the substrate, serves as a reference for the lattice planes of an epilayer of different isotopic composition. Employing XSW and photoemission, the position of the surface planes is determined from which the lattice constant difference $\Delta a$ is calculated. Scaled to $\Delta M=1 \mathrm{amu}$ we find $(\Delta a / a)$ of $-0.36 \times 10^{-5}$ and $-0.88 \times 10^{-5}$ for Ge and $-1.8 \times 10^{-5}$ and $-3.0 \times 10^{-5}$ for $\mathrm{Si}$ at 300 and $30 \mathrm{~K}$, respectively.
\end{abstract}

DOI: $10.1103 /$ PhysRevLett.86.5329

In recent years the study of the effect of the isotopic composition on various properties of materials such as the lattice dynamics and the electronic band structure of crystalline solids [1] has attracted increasing interest. The availability of highly isotopically enriched materials and promising technological applications is the main reason for the expanding research activities in this area. Practically all physical properties of crystals depend to some degree on their isotopic composition, sometimes yielding new, even exotic features. The thermal conductivity of isotopically enriched crystals, for example, can considerably exceed the values for the corresponding natural materials [2] and diamond made of isotopic pure ${ }^{13} \mathrm{C}$, should be harder than natural diamond [3] owing to the vibrational properties of isotopic pure ${ }^{13} \mathrm{C}$. A precise determination of the lattice constant of isotopically controlled $\mathrm{Si}$ is relevant, apart from basic interest, for its metrological applications in order to improve the accuracy in the determination of Avogadro's number [4] and thus establish an absolute standard of mass.

The influence of the isotopic composition on the lattice constant [5] is due to the combined effect of zero-point motion of the atoms and the anharmonicity of the potential. Thus, this is purely a quantum-mechanical effect. The resulting lattice constant difference, which is almost proportional to the relative mass difference $(\Delta M / M)$, is largest at $0 \mathrm{~K}$ and vanishes above the Debye temperature. Following the early work of London [5], few theoretical papers have appeared until recently on the issue of lattice constant versus isotopic mass [6-9]. Compared to the influence of the isotopic mass on the vibrational properties of crystals, the effect is small.

The change in lattice constant with isotopic mass is largest for crystals composed of light elements, because of the large relative mass change, and for crystals with high Debye temperature. Thus, the first $\mathrm{x}$-ray measurement was done on $\mathrm{LiF}$ [10] and not too long ago on diamond [11]. However, a change of only $\Delta a / a \approx 10^{-6}$ is expected for
PACS numbers: 68.49.Uv, 06.30.Dr, 68.60.-p, 81.05.Cy

heavier elements at room temperature for a $1 \%$ change of mass. Accurate measurements of such a small difference are not easy. Even if the effect can be measured by precision diffraction techniques, it is still expensive to grow a highly isotopically enriched bulk single crystal and it is difficult to do it with the necessary perfection. Thus, the results of an earlier study for the lattice constant difference between natural $\mathrm{Ge}$ and an isotopically enriched ${ }^{74} \mathrm{Ge}$ crystal using a modified diffraction technique [12] were later found to be in considerable disagreement with theoretical calculations [6]. Using a $1.36 \mu \mathrm{m}$ film of ${ }^{76} \mathrm{Ge}$ grown by molecular beam epitaxy on a natural Ge single crystal we demonstrated lately [13] that the x-ray standing wave (XSW) technique [14] can be used to determine the lattice constant difference with very high accuracy.

Here we report the application of the XSW method to the case of a highly enriched ${ }^{76} \mathrm{Ge}$ thin film on an almost isotopically pure ${ }^{70} \mathrm{Ge}$ crystal as well as to a ${ }^{30} \mathrm{Si}$ enriched film on a Si crystal with natural isotopic composition. The ${ }^{70} \mathrm{Ge}$ crystal was grown by the Bridgman technique with a moderate crystalline quality $\left(0.3^{\circ}\right.$ mosaic spread $)$ precluding standard XSW measurements. Details about this technique may be found in the literature [14-16] and we restrict us here to a brief description. An x-ray interference field is produced by a superposition of two coherent $\mathrm{x}$-ray plane waves. This $\mathrm{x}$-ray standing wave, generated, e.g., via Bragg diffraction by a substrate of a certain isotopic composition, exists above the substrate surface within the overlap region of the incident and reflected x-ray beams. The periodicity of the XSW matches exactly the periodicity of the diffraction planes of the substrate and thus can serve as a benchmark for the position of the atomic near-surface planes of an homoepitaxial overlayer with a different isotopic composition. These planes are displaced, the shift being caused by the cumulative effect of the lattice constant difference of the $N$ underlying lattice planes of the overlayer. Furthermore, for a pseudomorphic 
epitaxial layer the lattice mismatch in the direction normal to the surface will be enhanced [by a factor of 1.37 for $\mathrm{Ge}(111)$ and 1.44 for $\mathrm{Si}(111)$ [17] ] as determined by the Poisson ratio. Within the Bragg reflection region of the substrate, the position of the (antinodal) planes of the x-ray standing wave field can be changed (anti)parallel to the diffraction vector $\mathbf{H}$ by half of the XSW period by changing Bragg angle or energy. The position of the surface planes of the isotopically modified epilayer with respect to the host lattice is assessed by detecting the total photoelectron yield while scanning the Bragg reflection of the substrate [13].

When the dipole approximation is valid [18], and the photoemission process is exclusively initiated by the $\mathrm{x}$-ray interference field, the yield of photoelectrons excited by the XSW from near-surface atomic planes as a function of the glancing angle $\theta$ for $\sigma$ polarization is given by

$$
Y^{H}(\theta)=1+R(\theta)+\sqrt{R(\theta)} F^{H} \cos \left[v(\theta)-2 \pi P^{H}\right],
$$

where $R(\theta)$ is the reflectivity, $v(\theta)$ is the value of the phase of the reflected wave which changes by $\pi$ when passing the Bragg reflection region, and $F^{H}$ and $P^{H}$ are commonly called coherent fraction and coherent position, respectively. The coherent fraction describes the static and thermal displacements of the atoms from the mean position. The coherent position $P^{H}=\mathbf{H} \cdot r_{a}=z_{H} / d_{h k l}$. Here $z_{H}$ is the $z$ component of an atomic displacement $\mathbf{r}_{a}$ parallel to the diffraction vector $\mathbf{H}$ and describes the relative position of the atom normal to the diffraction planes with spacing $d_{h k l}$. For the ideal crystal $F^{H}=F_{I C}^{H}, P^{H}=P_{I C}^{H}$, and the photoelectron yield curve shows a characteristic shape. In the present cases, $P_{I C}^{H}=0$ and the yield curve exhibits a minimum and maximum intensity at the low- and high-angle sides of the reflectivity curve, respectively. For the mismatched epitaxial overlayer, the surface atoms are shifted by $N \times \Delta d$, where $\Delta d=d_{\text {substrate }}-d_{\text {overlayer, }}$, caused by the cumulative effect of the lattice constant difference of the $N$ lattice planes of the epilayer and thus $P^{H}=N \times(\Delta d / d)_{\perp}$. In the present XSW experiment the photoelectron yield is recorded as a function of glancing angle in the case of Si or energy in the case of Ge and $F^{H}$, $P^{H}$ are used as parameters for fitting the yield curves.

An isotopically enriched ${ }^{30} \mathrm{Si}$ film $\left(60 \%{ }^{30} \mathrm{Si}, 40 \%{ }^{28} \mathrm{Si}\right.$, $\bar{M}=29.20)$ with a thickness of $0.92 \mu \mathrm{m}$ was grown on a perfect single crystal $\mathrm{Si}(111)$ substrate with natural isotopic composition $\left(91 \%{ }^{28} \mathrm{Si}, 4 \%{ }^{29} \mathrm{Si}, 5 \%{ }^{30} \mathrm{Si}, \bar{M}=\right.$ 28.14) by liquid phase epitaxy (LPE). The isotopically enriched ${ }^{76} \mathrm{Ge}$ film $\left(86 \%{ }^{76} \mathrm{Ge}, 14 \%{ }^{74} \mathrm{Ge}, \bar{M}=75.72\right)$ with a thickness of $0.56 \pm 0.01 \mu \mathrm{m}$ was grown by molecular beam epitaxy (MBE) on the (111) surface of a Bridgman grown highly isotopically enriched ${ }^{70} \mathrm{Ge}$ single crystal $\left(96 \%{ }^{70} \mathrm{Ge}, 4 \%{ }^{72} \mathrm{Ge}, \bar{M}=70.08\right)$. Thickness and isotopic composition of the films had been determined with Rutherford backscattering spectrometry (RBS) and secondary ion mass spectrometry (SIMS), respectively.
The XSW measurements for ${ }^{76} \mathrm{Ge}$ on ${ }^{70} \mathrm{Ge}(111)$ were performed at the Hamburg Synchrotron Radiation Laboratory (HASYLAB) using $\mathrm{x}$ rays from the DORIS storage ring monochromatized by a $\mathrm{Si}(511) /(333)$ double-crystal monochromator with a bandpass of $0.3 \mathrm{eV}$ at an energy around $7.59 \mathrm{keV}$. The sample was mounted strain free on the cold finger of a He flow-through cryostat with a diode temperature sensor placed close by. The total yield of the emitted photoelectrons was detected with a channeltron within a range of grazing exit angle from $0^{\circ}$ to about $15^{\circ}$. The recorded electron signal originates from a specific depth which was taken into account in the analysis $[13,19]$. The Bragg angle of the $\mathrm{Si}(511) /(333)$ monochromator, and therefore the energy of the incident $x$ radiation was varied, thus scanning the Bragg reflection of the ${ }^{70} \mathrm{Ge}(111)$ substrate crystal. For Ge we chose the (444) reflection at a Bragg angle close to $90^{\circ}$. The ${ }^{70} \mathrm{Ge}(111)$ substrate crystal showed a moderate crystalline quality with a mosaic spread of $0.3^{\circ}$ which precludes standard XSW measurements. However, this did not compromise the accuracy of our measurements since the acceptance of $\mathrm{Ge}(444)$, i.e., the width of the single crystal reflection curve as calculated by the dynamical theory of x-ray diffraction [20], is in the backscattering geometry [21] wider than the $0.3^{\circ}$ mosaic spread. A sketch of the experimental setup at the RÖMO station of HASYLAB is presented in Fig. 1.

The X-ray standing wave measurements for the ${ }^{30} \mathrm{Si}$ film on natural $\mathrm{Si}(111)$ were carried out using $\mathrm{CuK} \alpha$ radiation from a stationary $x$-ray tube [13]. For these measurements the monochromator was scanned in angle passing the (333) Bragg reflection of the $\mathrm{Si}(111)$ single crystal substrate. The intensity of the reflected beam was monitored by a scintillation detector and used as a reference signal for drift correction during the measurements. With a typical photoelectron count rate of about $20 \mathrm{cps}$, the XSW measurement at a given temperature involved about 2000 scans and took about $12 \mathrm{~h}$.

The results of several XSW scans for ${ }^{76} \mathrm{Ge}$ on ${ }^{70} \mathrm{Ge}(111)$ and for ${ }^{30} \mathrm{Si}$ on natural $\mathrm{Si}(111)$ are shown in Figs. 2 and 3, respectively. A change of the shape of the photoelectron yield curves with temperature can be distinguished, which is quite drastic for Ge. The angular positions of maximum and minimum of the photoelectron yield change when lowering the temperature from 300 to $30 \mathrm{~K}$. This is due

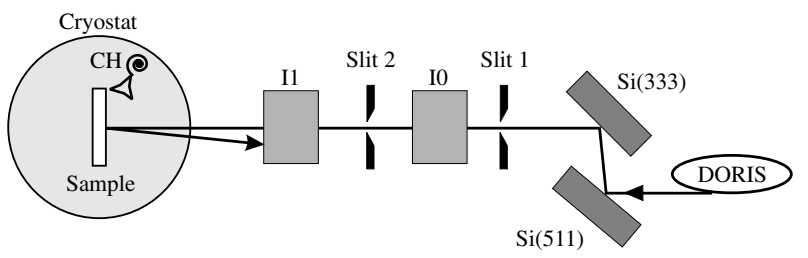

FIG. 1. Schematic experimental setup at the RÖMO beam line of HASYLAB with double crystal monochromator $\mathrm{Si}(511) /(333)$, slits, ionization chambers (I0, I1), channeltron $(\mathrm{CH})$, He flow-through cryostat, and the sample arranged in backscattering geometry. 


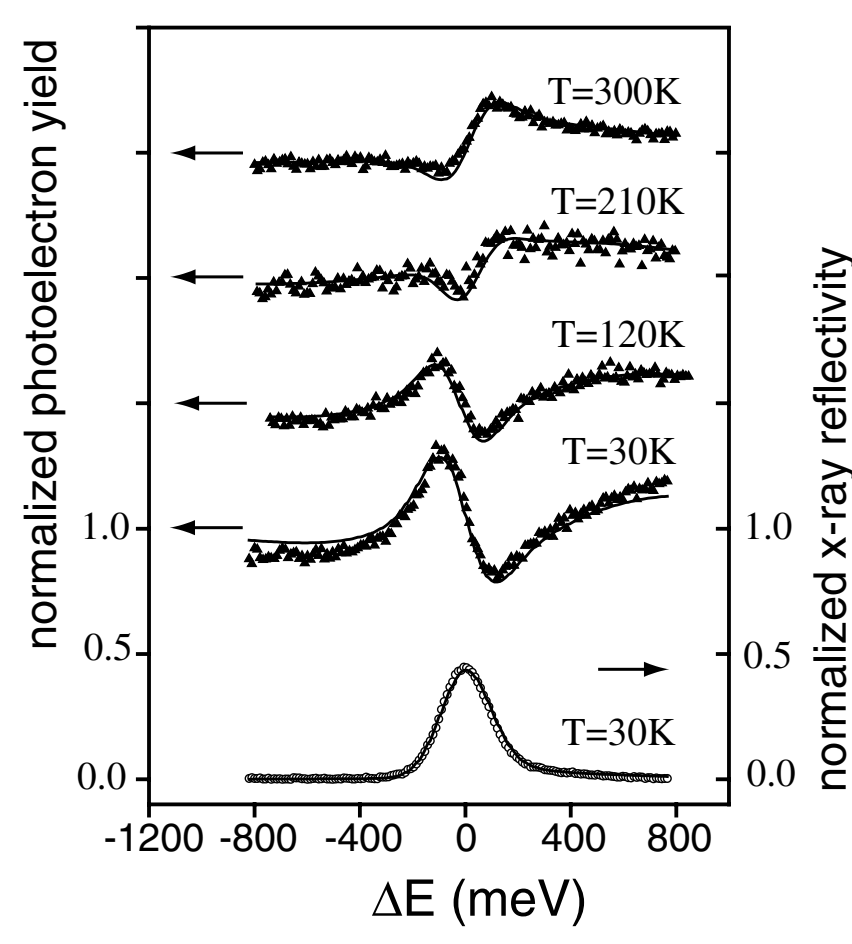

FIG. 2. X-ray reflectivity and photoelectron yield for different temperatures from a $0.56 \mu \mathrm{m}$ epitaxial ${ }^{76} \mathrm{Ge}$ layer on ${ }^{70} \mathrm{Ge}$ as a function of energy $\Delta E$ for the (444) reflection. The solid lines are fits to the experimental data (symbols).

to the shift of the surface planes by about the distance the wave field maxima traverse with angle, that is about $d_{444} / 2=40 \mathrm{pm}$ for Ge upon lowering the temperature to $30 \mathrm{~K}$, and clearly indicates the change in the lattice constant between the substrate and the epilayer when changing the temperature.

The results of best fits to the reflectivity and to the photoelectron yield are also shown in Figs. 2 and 3. The fit to the reflectivity determines the angular scale and fits to the photoelectron yield curves give the lattice mismatch $(\Delta d / d)_{\perp}$ from which the lattice constant differences are calculated [13]. By fitting the photoelectron yield curves for $30 \mathrm{~K}$ to the experimental data, the static Debye-Waller factor for the Ge homoepitaxial film was found to be unity and 0.55 for the Si LPE epilayer. These values were used for the further analysis. A possible reason for the relatively low value of the static Debye-Waller factor for the Si epilayer may be thickness fluctuations of the film of about $10 \%$ found by RBS. To calculate the lattice constant differences in the direction normal to the crystal surface for unstrained films, the elastic constants of $\mathrm{Ge}$ and $\mathrm{Si}$ have to be considered and the measured lattice mismatch has to be divided by a factor of 1.37 for Ge and 1.44 for Si [17] and these results are shown as a function of temperature in Fig. 4 for $\mathrm{Si}$ scaled to $\Delta M=1.00$. They are compared with theoretical calculations. Our data agree well with those of Herrero [9] within the limits of error. In contrast, the calculations of Biernacki and Scheffler [22] differ by about $30 \%$. It should be noted that

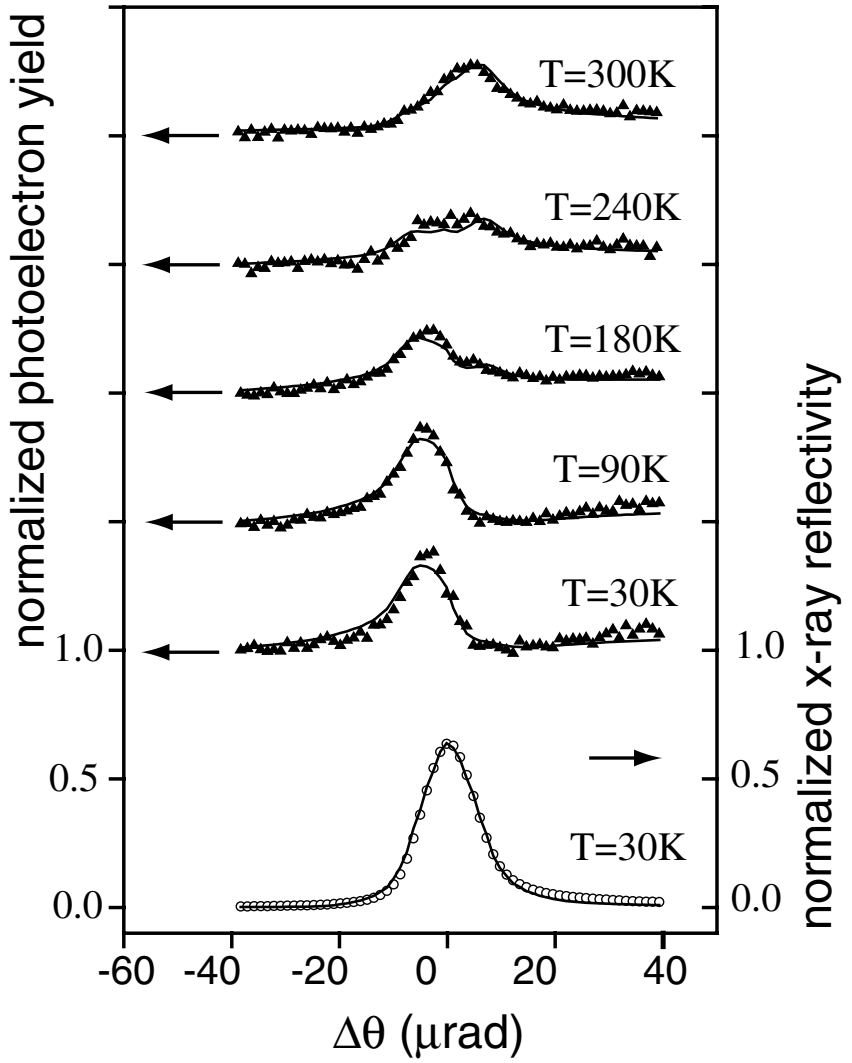

FIG. 3. X-ray reflectivity and photoelectron yield for different temperatures from a $0.92 \mu \mathrm{m}$ epitaxial ${ }^{30} \mathrm{Si}$ layer on natural $\mathrm{Si}$ as a function of glancing angle $\Delta \theta$ for the (333) reflection. The solid lines are fits to the experimental data (symbols).

the error in the values published by Herrero $(\sim 12 \%)$ is about twice as large as our experimental error, too large to demonstrate the anomaly below $150 \mathrm{~K}$ which is reflected

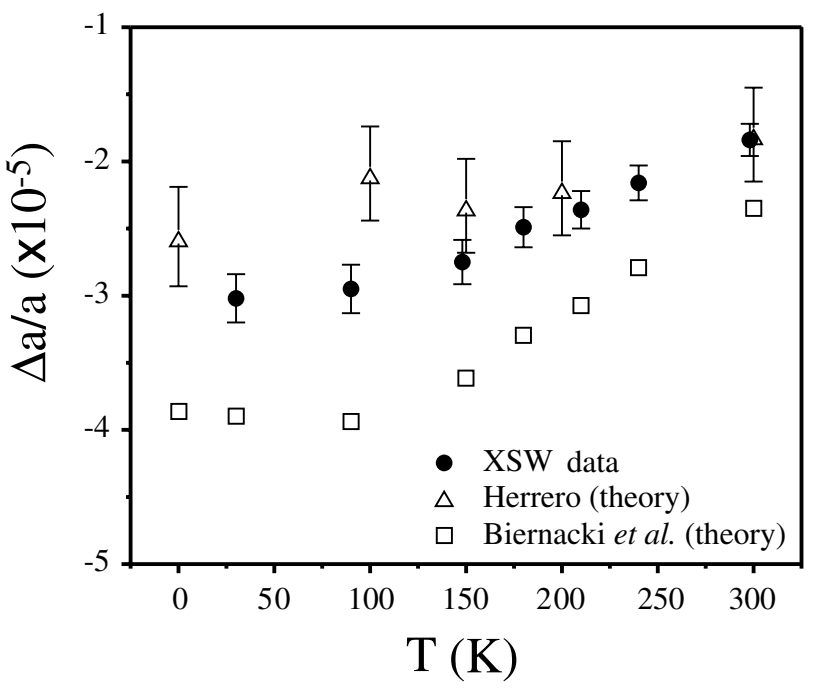

FIG. 4. The lattice constant difference of Si with isotopic mass as a function of temperature scaled to a mass difference of $\Delta M=1.00$. The XSW results (solid circles) are compared with calculations by Herrero [9] and Biernacki and Scheffler [22]. 
TABLE I. Lattice constant difference for Ge isotopes scaled to $\Delta M=1.00$.

\begin{tabular}{rcc}
\hline \hline $\mathrm{T}$ & ${ }^{76} \mathrm{Ge} /{ }^{70} \mathrm{Ge}$ & Ref. [13] \\
$(\mathrm{K})$ & $\Delta a / a\left(\times 10^{-6}\right)$ & $\Delta a / a\left(\times 10^{-6}\right)$ \\
\hline 30 & -8.8 & $\ldots$ \\
120 & -7.3 & -6.9 \\
210 & -4.8 & -4.8 \\
300 & -3.6 & -3.9 \\
& $\pm 5 \%$ & $\pm 7 \%$ \\
\hline \hline
\end{tabular}

in our experimental data and qualitatively shown in the calculation of Ref. [22].

The corresponding XSW results for Ge (also corrected with the help of the elastic constants [17]) are shown in Table I in comparison with our recently published XSW measurements [13]. Two of the listed data points have been generated by linearly interpolating the published data (over a range of about $10 \mathrm{~K}$ ) to match the temperatures of the present experiment. All lattice constant differences for $\mathrm{Ge}$ are scaled to $\Delta M=1.00$. For comparison with the measurements by Buschert et al. [12] and values calculated by Pavone and Baroni [6] and Noya et al. [8] we refer to Ref. [13]. The results of both sets of XSW measurements agree well with each other and provide strong support for the theoretical data by Pavone and Baroni [6].

In conclusion, we successfully applied the x-ray standing wave technique to measure with high accuracy the isotopic mass dependence of the lattice constants of Si and Ge. The XSW measurements were done using both a conventional $\mathrm{x}$-ray source for the study of ${ }^{30} \mathrm{Si}$ LPE film on natural $\mathrm{Si}(111)$ and synchrotron radiation in backscattering geometry for the analysis of ${ }^{76} \mathrm{Ge} \mathrm{MBE}$ film grown on a ${ }^{70} \mathrm{Ge}(111)$ crystal. Our technique requires crystals of only moderate crystalline quality and needs only miniscule amounts of isotopic material. Thus obtained results represent a stringent test for the theoretical calculations.

We would like to thank G. Bilger for the SIMS analysis of the thin films, E. Haller for providing the ${ }^{70} \mathrm{Ge}$ crystal, and D. Plachke for RBS thickness calibrations. Skillful technical assistance by W. Stiepany and support by the staff of HASYLAB and DESY are gratefully acknowledged. This work was supported by the German BMBF under Contract No. 05 SE8GUA 5.
*Permanent address: Space Material Science Research Center of the Institute of Crystallography, Russian Academy of Sciences, Kaluga 248640, Russia.

†Corresponding author.

Email address: zegenhagen@esrf.fr

[1] See, e.g., M. Cardona, in Advances in Solid State Physics, edited by R. Helbig (Vieweg, Braunschweig/Wiesbaden, Germany, 1994), Vol. 34, pp. 35-50.

[2] See, e.g., T. Ruf, R. W. Henn, M. Asen-Palmer, E. Gmelin, M. Cardona, H.-J. Pohl, G. G. Devyatych, and P.G. Sennikov, Solid State Commun. 115, 243 (2000), and references therein.

[3] A. K. Ramdas, S. Rodriguez, M. Grimsditch, T. R. Anthony, and W.F. Banholzer, Phys. Rev. Lett. 71, 189 (1993).

[4] See, e.g., P. Becker, H. Bettin, L. Koenders, J. Martin, A. Nicolaus, and S. Rottger, PTB Mitt. 106, 321 (1996).

[5] H. London, Z. Phys. Chem. 16, 302 (1958).

[6] P. Pavone and S. Baroni, Solid State Commun. 90, 295 (1994).

[7] A. Debernardi and M. Cardona, Phys. Rev. B 54, 11305 (1996)

[8] J. C. Noya, C. P. Herrero, and R. Ramirez, Phys. Rev. B 56, 237 (1997)

[9] C. P. Herrero, Solid State Commun. 110, 243 (1999).

[10] J. Thewlis, Acta Crystallogr. 8, 36 (1955).

[11] H. Holloway, K. C. Hass, M. A. Tamor, T. R. Anthony, and W. F. Banholzer, Phys. Rev. B 44, 7123 (1991).

[12] R. C. Buschert, A. E. Merlini, S. Pace, S. Rodriguez, and M. H. Grimsditch, Phys. Rev. B 38, 5219 (1988).

[13] A. Kazimirov, J. Zegenhagen, and M. Cardona, Science 282, 930 (1998).

[14] B. W. Batterman, Phys. Rev. A 133, 759 (1964).

[15] M. V. Koval'chuk and V. G. Kohn, Sov. Phys. Usp. 29, 426 (1986).

[16] J. Zegenhagen, Surf. Sci. Rep. 18, 199 (1993).

[17] J. Hornstra and W. J. Bartels, J. Cryst. Growth 44, 513 (1978).

[18] I. A. Vartanyants and J. Zegenhagen, Solid State Commun. 113, 299 (1999)

[19] M. V. Koval'chuk, V. G. Kohn, and E. F. Lobanovich, Sov. Phys. Solid State 27, 2034 (1985).

[20] M. von Laue, Röntgenstrahlinterferenzen (Akademische Verlagsgesellschaft, Frankfurt am Main, 1960), 3rd ed.

[21] K.-G. Huang, W. M. Gibson, and J. Zegenhagen, Phys. Rev. B 40, 4216 (1989); W. Graeff and G. Materlik, Nucl. Instrum. Methods 195, 97 (1982).

[22] S. Biernacki and M. Scheffler, J. Phys. Condens. Matter 6, 4879 (1994). 